\title{
On a Representation of the General Solution of a
} Functional-Differential Equation

\author{
M. Drakhlin and E. Litsyn
}

\begin{abstract}
A bstract. The general solution of a functional-differential equation with non-Volterra operator is found by its reducing to an infinite system. An integral representation of the general solution of this system is presented. Properties of the kernel of this system are studied.
\end{abstract}

Keywords: Functional-differential equations, superposition operators, Volterma operators AMS subject classiflcation: $34 \mathrm{~K} 05,47 \mathrm{~B} 38$

Consider the quasi-linear functional-differential equation

$$
\mathcal{L} x=F x
$$

where $\mathcal{L}$ is a linear functional-differential operator and $F$ is a nonlinear superposition operator. As the most relevant example of equation (1) we mention the equation

$$
\begin{aligned}
\left(\mathcal{L}_{0} x\right)(t) \equiv \dot{x}(t)+B(t) \dot{x}(g(t))+A(t) x(h(t)) & =f(t, x(t)) & & (t \in[0, \infty)) \\
\dot{x}(\xi)=x(\xi) & =0 & & (\xi<0) .
\end{aligned}
$$

Here $x(t) \in \mathbb{R}^{n}, t \in[0, \infty)$, and $A(t), B(t)$ are $n \times n$-matrices whose entries are measurable essentially bounded real functions on the half-line. The functions $g, h:[0, \infty) \rightarrow \mathbb{R}$ are measurable, and the function $f:(0, \infty) \times \mathbb{R}^{n} \rightarrow \mathbb{R}^{n}$ is locally summable:

Assume that $g$ has the property that, for all measurable subsets $e \subset[0, \infty)$,

$$
m(e)=0 \text { implies } m\left(g^{-1}(e)\right)=0
$$

where $m$ is the Lebesgue measure. It is well known that condition (3) is necessary and sufficient for the following implication: if a function $z:(-\infty, \infty) \rightarrow \mathbb{R}^{n}$ is measurable, then the superposition $z(g):[0, \infty) \rightarrow \mathbb{R}^{n}$ is also measurable.

Denote by $D_{[a, b]}$ the space of all functions $x:[a, b] \rightarrow \mathbb{R}^{n}$ for which the norm

$$
\|x\|_{D_{(e, 0]}}=\|x\|_{C_{[\bullet, b]}}+\|\dot{x}\|_{L_{[a, b]}}
$$

M. Drakhlin: College of Judea and Samaria, Math. Res. Inst., A riel 44820, Israel

E. Litsyn: Bar-Ilan University, Dep. Math., Ramat - Gan 52900, Israel

This research was supported by the Ministry of Science and Technology of the State of Israel 
is finite; here $L_{[a, b]}$ is the space of summable functions $x:[a, b] \rightarrow \mathbb{R}^{n}$. Similarly, $D_{[0, \infty)}$ and $L_{(0, \infty)}$ are the spaces of locally absolutely continuous and summable functions, respectively. It is possible to define a topology in these spaces by a countable system of semi-norms

$$
\|x\|_{D_{(0, \infty)}}^{(\beta)}=\|x\|_{D_{[0,0]}} \quad \text { and } \quad\|x\|_{L_{[0, \infty)}}^{(\beta)}=\|x\|_{L_{[0, \rho]}} \quad(\beta \in \mathbb{N}) .
$$

In the study of equation (1), two main different cases have to be distinguished.

Case 1: The Operator $\mathcal{L}_{0}$ is Volterra. In this case it is enough to require

$$
g(t) \leq t \quad \text { and } \quad h(t) \leq t \quad(t \in[0, \infty)) .
$$

Then, the conditions for representing the solution of the (linear) problem

$$
\left(\mathcal{L}_{0} x\right)(t)=f(t) \quad(t \in[0, \infty)) \quad \text { and } \quad x(0)=0
$$

in the form

$$
x(t)=\int_{0}^{t} C(t, s) f(s) d s
$$

are well-known. The substitution

$$
x(t)=(W f)(t) \equiv \int_{0}^{t} C(t, s) f(s) d s
$$

reduces problem (1) to the equation

$$
f(t)=(F W f)(t) \quad(t \in[0, \infty))
$$

in the space $L_{(0, \infty)}$. The existence of the Cauchy matrix $C(t, s)$ and its properties have found a great deal of attention in many papers and monographs (see $[1,4]$ and references there). The neutral-type equations were studied in detail in [2].

Case 2: The Operator $\mathcal{L}_{0}$ is Non-Volterra. Assume, for example, that

$$
g(t) \leq t+1 \quad \text { and } \quad h(t) \leq t+1 \quad(t \in[0, \infty)) .
$$

In this case the solution of problem (5) is in general not representable in the form (6). So, the question about representing the general solution of the equation

$$
\left(\mathcal{L}_{0} x\right)(t)=f(t) \quad(t \in[0, \infty))
$$

becomes relevant. We will try to obtain an integral representation for the solution of equation (10) by reducing it to a countable system of functional-differential equations. Denote,

$$
x_{i}^{*}(t)=\chi_{\{i-1, i)}(t) x(t) \quad(t \in[0, \infty), i \in \mathbb{N})
$$


where $\chi_{(i-1, i)}$ is the characteristic function of the interval $[i-1, i)$. Since $x(t)=$ $\sum_{i=1}^{\infty} x_{i}^{*}(t)$, equation (10) takes then the form

$$
\begin{aligned}
\sum_{i=1}^{\infty} \dot{x}_{i}^{*}(t)+B(t) \sum_{i=1}^{\infty} \dot{x}_{i}^{*}(g(t))+\dot{A(t)} \sum_{i=1}^{\infty} x_{i}^{*}(h(t)) & =f(t) & & (t \in[0, \infty)) \\
(\dot{x})_{i}^{*}(\xi)=x_{i}^{*}(\xi) & =0 & & (\xi<0 ; i \in \mathbb{N}) .
\end{aligned}
$$

Furthermore, assume that $m\left(h^{-1}(i)\right)=0$ for $i \in \mathbb{N}$. From (9) we get

$$
\begin{aligned}
\dot{x}_{i}^{*}(t)+B(t) \sum_{k=1}^{i+1} \dot{x}_{k}^{*}(g(t))+A(t) \sum_{k=1}^{i+1} x_{k}^{*}(h(t)) & =f(t) & & (t \in[i-1,1]) \\
\dot{x}_{i}^{*}(\xi)=x_{i}(\xi) & =0 & & (\xi<0 ; i \in \mathbb{N}) .
\end{aligned}
$$

For $\tau \in[0,1]$ and $i \in \mathbb{N}$, set

$$
\begin{array}{lll}
x_{i}(\tau)=x_{i}^{*}(i-1+\tau), & B_{i}(\tau)=B(i-1+\tau), & A_{i}(\tau)=A(i-1+\tau) \\
f_{i}(\tau)=f(i-1+\tau), & g_{i}(\tau)=g(i-1+\tau), & h_{i}(\tau)=h(i-1+\tau) .
\end{array}
$$

In this notation we get the following system of equations $(\tau \in[0,1])$ :

$$
\begin{aligned}
\left(\mathcal{L}_{i} x\right)(\tau) & \equiv \dot{x}_{i}(\tau)+B_{i}(\tau) \sum_{k=1}^{i+1} \dot{x}_{k}\left(g_{i}(\tau)\right)+A_{i}(\tau) \sum_{k=1}^{i+1} x_{k}\left(h_{i}(\tau)\right)=f_{i}(\tau) \\
\dot{x}_{k}(\xi) & =x_{k}(\xi)=0 \quad(\xi \in(-\infty, 0) \cup(1, \infty) ; k=1,2, \ldots, i+1 ; i \in \mathbb{N}) .
\end{aligned}
$$

Now, if $x_{1}, x_{2}, \ldots$ solve the problem (13) with boundary conditions

$$
x_{1}(0)=\alpha \quad\left(\alpha \in \mathbb{R}^{n}\right) \quad \text { and } \quad x_{i}(0)=x_{i-1}(1) \quad(i=2,3 ; \ldots),
$$

then the function

$$
x(t)=x_{i}(t-i+1) \quad(t \in[i-1, i])
$$

solves the problem (10) with initial condition $x(0)=\alpha$. The last equality along with our study of boundary value problems for infinite systems of functional-differential equations [5] leads to the following

Lemma 1. Let the problem

$$
\left(\mathcal{L}_{i} x\right)(t)=f_{i}(\tau) \quad \text { and } \quad x_{i}(0)=\alpha_{i} \quad(\tau \in[0,1] ; i \in \mathbb{N})
$$

be uniquely solvable for every $\alpha_{i} \in \mathbb{R}^{n}$ and $f_{i} \in L_{[0,1]}(i \in \mathbb{N})$. Then the general solution of equation (10) has a representation

$$
x(t)=X_{i}(t-i+1) c+\int_{0}^{1} G_{i}(t-i+1, s) f(s) d s \quad(t \in[i-1, i])
$$


where $c=(\alpha, x(0), x(1), \ldots) \in \mathbb{R}^{\infty}$ and $f=\left(f_{1}, f_{2}, \ldots\right)$. Here $X_{i}$ is the $i$-th section of the infinite fundamental matrix $X$ for the system (19), and $G_{i}$ is the $i$-th section of the infinite Green matrix $G$ of the preceding system.

In [5] it is shown that, under conditions of Lemma 1, the general solution $y=$ $\left(x_{1}, x_{2}, \ldots\right)$ for the system (13) admits a representation

$$
y(\tau)=X(\tau) c+\int_{0}^{1} G(\tau, s) z(s) d s
$$

Here $X$ is the infinite fundamental matrix of (13), and $G$ is the infinite Green matrix of the solution of (13), subject to the conditions

$$
x_{1}(0)=\alpha \quad \text { and } \quad x_{i+1}(0)-x_{i}(1)=0 \quad(i \in N) .
$$

Let us study the properties of the infinite Green matrix for the system (13) from a more general viewpoint. Consider an infinite system of linear functional-differential equations

$$
M x=f,
$$

where $M: D_{[0,1]}^{\infty} \rightarrow L_{[0,1]}^{\infty}$ is a linear bounded operator. Here $D_{[0,1]}^{\infty}$ and $L_{[0,1]}^{\infty}$ are the spaces of functions $x=\left(x_{1}, x_{2}, \ldots\right):[0,1] \rightarrow R^{\infty}$ with absolutely continuous and summable on $[0,1]$ components, respectively. Denote by $D_{[0,1]}^{\beta}$ and $L_{[0,1]}^{\beta}(\beta=$ $0,1, \ldots)$ the spaces of absolutely continuous and summable $\beta$-dimensional functions $x^{\beta}=\left(x_{1}, x_{2}, \ldots, x_{\beta}\right):[0,1] \rightarrow \mathbb{R}^{\beta}$, respectively. Let $K_{\beta}$ denote the projection of a vector $x=\left(x_{1}, x_{2}, \ldots\right)$ with an infinite number of components to the vector $x^{\beta}=$ $\left(x_{1}, x_{2}, \ldots, x_{\beta}\right)$ consisting of the first $\beta$ components of the vector $x$; thus, $x^{\beta}=K_{\beta} x$. A system of semi-norms in $D_{[0,1]}^{\infty}$ and $L_{[0,1]}^{\infty}$ is defined by the equalities

$$
\|x\|_{D_{[0,1]}^{\infty}}^{(\beta)}=\left\|K_{\beta} x\right\|_{D_{[0,1]}^{\rho}} \quad \text { and } \quad\|x\|_{L_{[0,1]}^{\infty}}^{(\beta)}=\left\|K_{\beta} x\right\|_{L_{[0,1]}^{\rho}} \quad(\beta \in \mathbb{N})
$$

Let us give several definitions, which are necessary for what follows, from the theory of linear equations in Fréchet spaces (see [5]).

Let $E$ and $\mathcal{E}$ be complete countably normed spaces with.systems of semi-norms $\|\cdot\|_{E}^{(\alpha)}$ and $\|\cdot\|_{\mathcal{E}}^{(\beta)}(\alpha, \beta \in \mathbb{N})$, respectively. A linear operator $T: E \rightarrow \mathcal{E}$ is said to have the $V_{\phi}$ property $\left(T \in V_{\phi}\right)$ if for every natural $\beta$ there exists a natural number $\phi(\beta)$ such that the equality $\|\xi\|_{E}^{(\phi(\beta))}=0$ implies the equality $\|T \xi\|_{\mathcal{E}}^{(\beta)}=0$. Evidently, any bounded operator $T: E \rightarrow \mathcal{E}$ possesses this property.

Two elements $\xi, \eta \in E$ are said to be $\alpha$-equivalent if they coincide in the $\alpha$-seminorm, i.e. $\|\xi-\eta\|_{E}^{(\alpha)}=0$. Identifying $\alpha$-equivalent elements, we get a Banach space $E^{\alpha}$ of elements $\xi^{\alpha}$, where the norm is defined by the $\alpha$ - semi-norm of the space $E$.

Given $T \in V_{\phi}$, for every $\beta \in \mathbb{N}$ one can define a linear operator $T_{\beta}: E^{\phi(\beta)} \rightarrow \mathcal{E}^{\beta}$ by putting

$$
T_{\beta} x=(T \xi)^{\beta} \quad\left(x=\xi^{\phi(\beta)} ; \xi \in E\right) .
$$


The space of sequences $g=\left(g_{\lambda_{1}}, g_{\lambda_{2}}, \ldots\right)$ of linear bounded functionals $g_{\lambda_{4}}: E^{\lambda_{4}} \rightarrow$ $\mathbb{R}\left(g_{\lambda_{k}} \in\left[E^{\lambda_{1}}\right]^{*}\right)$ is said to be $F_{\lambda^{-a d j o i n t}}$ to the space $E$ and is denoted by $E_{\lambda}^{\prime}(\lambda=$ $\left.\left(\lambda_{1}, \lambda_{2}, \ldots\right)\right)$. In the case of $\lambda_{k}=k(k \in \mathbb{N})$ we will skip the index.

For $k \in \mathbb{N}$, denote by $T_{k}^{*}:\left[\mathcal{E}^{k}\right]^{*} \rightarrow\left[E^{\phi(k)}\right]^{*}$ the adjoint operator to $T_{k}: E^{\phi(k)} \rightarrow \mathcal{E}^{k}$. The operator $T^{\prime}=\left(T_{1}^{*}, T_{2}^{*}, \ldots\right): \mathcal{E}^{\prime} \rightarrow E_{\lambda}^{\prime}, \lambda_{k}=\phi(k)$ is then called the $V_{\phi}$-adjoint to

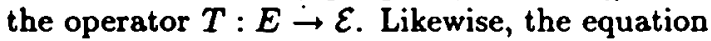

$$
T^{\prime} g=f \quad\left(g \in \mathcal{E}^{\prime}, f \in E_{\lambda}^{\prime} ; \lambda_{k}=\phi(k), k \in \mathbb{N}\right)
$$

is called $V_{\phi}$-adjoint to the equation $T \xi=\eta$.

We return now to equation (15). Let $M \in V_{\phi}$. Since $x(t)=\int_{0}^{t} \dot{x}(s) d s+x(0)(t \in$ $[0,1])$, equation (15) is reducible to the form

$$
(Q \dot{x})(t)+P(t) x(0)=f(t) \quad(t \in[0,1])
$$

where $Q=M V,(V y)(t)=\int_{0}^{t} y(s) d s$ and $P$ is an infinite matrix whose entries are essentially bounded functions on $[0,1]$. If the problem

$$
(M x)(t)=f(t), \quad x(0)=0
$$

is uniquely solvable for every $f \in L_{[0,1]}^{\infty}$, then by virtue of [5: Theorem 7] we have $x(t)=\int_{0}^{1} G(t, s) f(s) d s$. Applying the Green operator to both sides of $(Q \dot{x})(t)=f(t)$ we get

$$
\int_{0}^{1} G(t, s)(Q \dot{x})(s) d s=\int_{0}^{1} \chi[0, t](s)(I \dot{x})(s) d s .
$$

Hence, for every $t \in[0,1]$ and $\alpha \in \mathbb{N}$ we conclude that

$$
\begin{aligned}
\int_{0}^{1} G_{\alpha \psi(\alpha)}(t, s) K_{\psi(\alpha)}\left[L_{[0,1]}^{\infty}\right](Q \dot{x})(s) d s \\
\quad=\int_{0}^{1} G_{\alpha \psi(\alpha)}(t, s)\left(Q_{\psi(\alpha)} \dot{x}^{\phi(\psi(\alpha))}\right)(s) d s \\
=\int_{0}^{1} \chi_{[0, t]}(s) I_{\alpha \phi(\psi(\alpha))} K_{\phi(\psi(\alpha))} \dot{x}(s) d s
\end{aligned}
$$

where $I_{\alpha \phi(\psi(\alpha))}$ is the result of adding $[\phi(\psi(\alpha))-\alpha]$ zero columns to the identity matrix

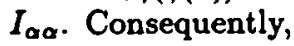

$$
\left[Q_{\psi(\alpha)}^{*} G_{\alpha \psi(\alpha)}(t, \cdot)\right](s)=\chi[0, t](s) I_{\alpha \alpha} \quad(t \in[0,1], \alpha \in \mathbb{N})
$$

Thus, for every $t \in[0,1]$ the matrix $G(t, \cdot)$ satisfies the $V_{\phi}$-adjoint equation in the second argument. We summarize with the following 
Theorem 1. For every $t \in[0,1]$ the Green matrix for the problem (16) satisfies the matrix equation in the second argument $\left[Q^{\prime} G(t, \cdot)\right](s)=\chi[0, t](s) I(s \in[0,1])$ where $I$ is the infinite identity matrix.

When applying the fundamental principles of both linear and nonlinear functional analysis to functional-differential equations, one often has to require also the compactness (or weak compactness) of the operators involved, rather than just their continuity. An operator $T: E \rightarrow \mathcal{E}, T \in V_{\phi}$ is called $V$-completely continuous (respectively, weakly $V$-completely continuous) if the operators $T_{\beta}: E^{\phi(\beta)} \rightarrow \mathcal{E}^{\beta}$ are completely continuous (respectively, weakly completely continuous) for every natural $\beta$. The proof of the following theorem is straightforward (see [6]).

Theorem 2. Let $Q=J-K$, where $K: L_{[0,1]}^{\infty} \rightarrow L_{[0,1]}^{\infty}$ is a weakly V-completely continuous operator. Then the following holds:

1. For every $s \in[0,1], G(\cdot, s)$ is absolutely continuous on $[0, s) \cup(s, 1]$, and

$$
G(s+0, s)-G(s-0, s)=I \text {. }
$$

2. For every $z \in L_{[0,1]}^{\infty}$, the equality

$$
\frac{d}{d t} \int_{0}^{1} G(t, s) z(s) d s=z(t)+\int_{0}^{1} \frac{\partial}{\partial t} G(t, s) z(s) d s
$$

holds.

9. For every $s \in[0,1], G(\cdot, s)$ satisfies the relation

$$
\frac{\partial}{\partial t} G(t, s)-\int_{0}^{1} K(t, \tau) \frac{\partial}{\partial \tau} G(\tau, s) d \tau+P(t) G(0, s)=K(t, s) .
$$

\section{References}

[1] Azbelev, N., Maksimov, V. and L. Rakhmatullina, L.: Introduction to the Theory of Functional-Differential Equations (in Russian). Moscow: Nauka 1991.

[2] Drakhlin, M.: On oscillatory properties of some functional-differential equations (in Russian). Diff. Uravn. 22 (1986), 396 - 402; Engl. transl.: Diff. Equ. 22(1986), 283 289.

[3] Drakhlin, M.: Some problems in stability of neutral-type functional-differential equations (in Russian). Diff. Uravn. 22 (1986), 925. Engl. transl.: Diff. Equ. 22 (1986), 919 - 925.

[4] Hale, J.: Theory of Functional-Differential Equations. Berlin: Springer - Verlag 1977.

[5] Litsyn, E.: General theory of functional-differential equations (in Russian). Diff. Uravn. 24 (1988), 977 - 986; Engl. transl.: Diff. Equ. 24 (1988), 638 - 646.

[6] Maksimov, V.: On the Cauchy formula for functional-differential equations (in Russian). Diff. Uravn. 13 (1977), 601 - 606; Engl. transl.: Diff. Equ. 13 (1977), 405 - 409.

Received 09.08.1993 